seizure period. In contrast, the CPT group has the shortest active seizure period, but often presents with SE. The most characteristic clinical manifestation, occurring in all patients, is the ictal vomiting. In PS a genetic predisposition to seizures is common. The outlook is generally good, with seizure remission before 12 years of age.

Abnormal neuroimaging in patients with BECTS is reported in $15 \%$ of 71 cases (Gelisse P, Corda D, Raybaud C, et al. Epilepsia 2003;44:372-378). These included dilated lateral ventricles, neonatal intraventricular hemorrhage, and agenesis of corpus callosum.

\title{
EFFECT OF METHYLPHENIDATE IN ADHD WITH EPILEPSY OR WITH EEG ABNORMALITIES WITHOUT SEIZURES
}

The safety and efficacy of methylphenidate (MPH) in the treatment of ADHD, group 1) with epilepsy and group 2) with EEG abnormalities without seizures, were determined in a study of 119 children ( 98 males, 21 females; ages 6 to 16 years) at Gazi University, Ankara, Turkey. The neurologic examination showed only soft signs in $8 \%$ of patients. Brain scans, vision and hearing were normal, and WISC-R scores were average (mean $92.3+/-0.2$ ). Of 57 patients with epilepsy, 34 had complex partial seizures, $13 \mathrm{had}$ tonic-clonic seizures, and 10 had multiple seizures; 52 received antiepileptic (AED) monotherapy and 5 polytherapy. At the end of the 12 month period of AED with added MPH $(0.3-1 \mathrm{mg} / \mathrm{kg} /$ day $)$ treatment, the mean yearly seizure frequency was unchanged $(8.2$ pre-MPH treatment and 8.1 post-treatment), but $5(10 \%)$ patients had an increase in seizures. None of the 62 patients with ADHD and EEG abnormalities developed seizures during treatment with MPH (without AED). Percentage of patients with EEG epileptiform activity was decreased in both the ADHD+epilepsy group and the ADHD without seizure group; $35.1 \%$ at baseline of $19.3 \%$ post-treatment $(\mathrm{p}=0.01)$ and $24.2 \% \mathrm{cf} 12.9 \%(\mathrm{p}=0.02)$, respectively. The baseline and the last mean total ADHD and Conners' scores from parents' and teachers' questionnaires showed significant improvements in both the epileptic and the abnormal EEG groups. On clinician ratings, 92 of the 119 children in the study showed reduced ADHD symptoms, and only 9 patients had deterioration in behavior, 6 of whom were epileptic. Side effects of MPH were observed in $26(22 \%)$ patients and included loss of appetite (23), sleep disorder (19), stomachache (14), headache (14), and motor tics (2); none required MPH withdrawal. MPH is considered safe and effective in patients with ADHD and epilepsy, when combined with AED treatment, and in patients with ADHD and abnormal EEG without seizures. (Gucuyener K, Erdemoglu AK, Senoi S, et al. Use of methylphenidate for attention-deficit hyperactivity disorder in patients with epilepsy or electroencephalographic abnormalities. J Child Neurol 2003;18:100-112). (Dr A Kemal Erdemoglu, Cinnah Cad Kirkpinar sok 12/4 Cankaya, Ankara 06690, Turkey).

COMMENT. The PDR advises against the use of methylphenidate (MPH) in patients with seizures or with abnormal EEG. MPH is thought to lower the convulsive threshold. The present report confirms that of previous authors, and shows that MPH may be a safe and effective treatment for ADHD complicated by epilepsy, provided that seizures are controlled by anticonvulsant medication (In Millichap JG. Attention Deficit Hyperactivity and Learning Disorders. PNB Publishers, 1998;pp179-181). MPH should be discontinued or the dosage reduced, if seizures recur despite optimal doses of AED. The 
present study finds that the likelihood of seizure recurrence is $10 \%$ in ADHD patients with treated epilepsy who receive MPH. In ADHD patients with abnormal EEG without clinical seizures, MPH did not appear to cause seizures and may have a normalizing effect on the EEG (see also Swartwood MO et al. Pediatr Neurol 1998;18:244-250). The prevalence of abnormal EEG in patients with ADHD is reviewed in Ped Neur Briefs Oct 2000;14:73-74.

Hemmer SA, Pasternak JK, et al found that $15.4 \%$ of nonepileptic children with ADHD demonstrated epileptiform abnormalities in the EEG (Pediatr Neurol 2001;24:99102). During treatment with MPH, seizures occurred in 1 of 175 nonepileptic ADHD patients with normal EEG (0.6\%), and in 3 of 30 with epileptiform EEGs $(10 \%)$. Of 12 children with rolandic spikes, $2(16.7 \%)$ developed seizures with MPH. A normal EEG indicates minimal risk of seizures with MPH therapy for ADHD. In contrast, an epileptiform EEG in nonepileptiform children with ADHD predicts a $10-17 \%$ risk of occurrence of seizures. The prophylactic value of AED treatment in these ADHD patients must be weighed against the potential adverse effects of AED, especially on cognition. My own present policy is to withhold AED "umbrella" therapy with MPH. Atomoxetine (Strattera), the new nonstimulant treatment for ADHD, may prove advantageous in these cases, but its effect on the EEG remains to be established.

\section{CLASSIFICATION, DIAGNOSIS, AND TREATMENT OF SYNCOPE}

The classification, diagnosis, investigation, and management of syncopal attacks in childhood are reviewed from the Department of Cardiology, Royal Hospital for Sick Children, Glasgow, UK. The causes of childhood syncope are classified in three groups: neurally mediated, cardiovascular, and noncardiovascular pseudo-syncope. Neurallymediated (neurocardiogenic) syncopes are most commonly reflex in type, and consist of a transient disturbance in the autonomic control of heart rate and blood pressure. They occur at any age, typically in toddlers and in children between 9 and 14 years of age. Characteristically, the child is either sitting or standing, and suffers loss of tone and consciousness, preceded by dizziness, nausea, and pallor. Hypotension and/or bradycardia result in cerebral hypoxia, and when severe, an anoxic seizure and incontinence. The reflex anoxic seizure resembles a pallid breath-holding attack in a toddler, and consists of opisthotonus and twitching. Unlike an epileptiform discharge, the electroencephalographic (EEG) record is flat during a reflex anoxic seizure.

The diagnosis may be made by a detailed and careful history, and investigations are usually normal. For recurrent syncope, a 12 lead electrocardiogram is advised, primarily to exclude a long QT interval. "Warning bells" that prompt investigation include syncope in response to fright or exercise, syncope while supine, and a family history of sudden unexplained death. Tests such as an echocardiogram or EEG are usually not indicated in a single or uncomplicated attack; they should be considered principally in patients with other cardiac or neurologic symptoms and signs. A tilt test is positive in approximately $50 \%$ of children age 6 years or older with recurrent neurally-mediated syncope, and cardiac event monitoring can be a productive investigation in the toddler age group with reflex asystolic syncope. In psychogenic pseudosyncope, the ECG, EEG, and blood pressure are normal during continuous ambulatory inpatient monitoring. Management of neurally-mediated syncope consists of reassurance, plenty of fluids and added salt, and maintenance of blood pressure by crossing legs and folding the arms. Drug therapy is considered when simple 